\title{
Convergence of moments for Axiom A and nonuniformly hyperbolic flows
}

\author{
Ian Melbourne $^{*} \quad$ Andrei Török ${ }^{\dagger}$ \\ 11 October, 2010; revised 10 February 2011
}

\begin{abstract}
In the paper, we prove convergence of moments of all orders for Axiom A diffeomorphisms and flows. The same results hold for nonuniformly hyperbolic diffeomorphisms and flows modelled by Young towers with superpolynomial tails. For polynomial tails, we prove convergence of moments up to a certain order, and give examples where moments diverge when this order is exceeded.

Nonuniformly hyperbolic systems covered by our result include Hénon-like attractors, Lorenz attractors, semidispersing billiards, finite horizon planar periodic Lorentz gases, and Pomeau-Manneville intermittency maps.
\end{abstract}

\section{Introduction}

It is well-known that Axiom A diffeomorphisms and flows enjoy strong statistical properties such as the central limit theorem (CLT) for Hölder observables [8, 36, 39, 34]. Specifically, let $\Lambda$ be a nontrivial hyperbolic basic set for an Axiom A diffeomorphism $f$ with equilibrium measure $\mu$ corresponding to a Hölder potential. If $v: \Lambda \rightarrow \mathbb{R}$ is a Hölder observable with $\int_{\Lambda} v d \mu=0$, then the normalised Birkhoff sum $n^{-\frac{1}{2}} \sum_{j=0}^{n-1} v \circ f^{j}$ converges in distribution to a normal distribution with mean zero and variance $\sigma^{2} \geq 0$. Moreover, either $\sum_{j=0}^{n-1} v \circ f^{j}$ is uniformly bounded or $\sigma^{2}>0$, and the latter is the typical case.

*Department of Mathematics, University of Surrey, Guildford, Surrey GU2 7XH, UK. ism@math.uh.edu

${ }^{\dagger}$ Department of Mathematics, University of Houston, Houston, TX 77204-3008, USA, and Institute of Mathematics of the Romanian Academy, P.O. Box 1-764, RO-70700 Bucharest, Romania. torok@math.uh.edu 
The analogous result for Axiom A flows (with Birkhoff sums replaced by integrals) was proved by Ratner [34] (an unnecessary mixing assumption was removed in [29]). However, there is a lack of symmetry concerning the formula for $\sigma^{2}$. In the diffeomorphism case, $f: \Lambda \rightarrow \Lambda$ has exponential decay of correlations (up to a finite cycle) for Hölder observables and a consequence is existence of the limit

$$
\sigma^{2}=\lim _{n \rightarrow \infty} \int_{\Lambda}\left(n^{-\frac{1}{2}} \sum_{j=0}^{n-1} v \circ f^{j}\right)^{2} d \mu .
$$

This is a result on convergence of second moments. Convergence in distribution to a normal distribution with this variance is proved as a second step. In contrast, Axiom A flows need not be mixing, and even when they are mixing the decay of correlations can be arbitrarily slow [32]. The approach of Ratner [34] is to pass to the Poincaré map and to derive the CLT for the flow from the CLT for diffeomorphisms, thus bypassing mixing properties and convergence of moments. Convergence of second moments for Axiom A flows was proved recently in McMullen [25].

In this paper, we give a different proof from [25] which yields convergence of moments of all orders for Axiom A diffeomorphisms and flows. Moreover, our result extends to nonuniformly hyperbolic maps and flows that can be modelled by Young towers with exponential tails [42]. This includes Hénon-like attractors, semidispersing billiards (finite and infinite horizon), finite horizon planar periodic Lorentz gases, Lorentz gases with cusps, and geometric Lorenz attractors.

For systems with polynomial decay of correlations modelled by Young towers with polynomial tails [43], there are restrictions on the order of the moments that can converge. If the Young tower has decay of correlations $n^{-\beta}, \beta>1$, then we prove convergence of $q$ th moments for $q<2 \beta$ (for maps and the associated flows) and give examples where $q$ th moments diverge for $q>2 \beta$.

Remark 1.1 Our results are restricted to systems modelled by Young towers with summable decay of correlations $(\beta>1)$ where the CLT holds with the standard $n^{\frac{1}{2}}$ normalisation (or $t^{\frac{1}{2}}$ in the case of flows). For the infinite horizon planar periodic Lorentz gas, Szász \& Varjú [40] proved a nonstandard CLT with normalisation $(t \log t)^{\frac{1}{2}}$. In this situation convergence of moments seems to be more subtle, see for example $[2,16]$.

The remainder of this paper is organised as follows. In Section 2, we prove a result about convergence of moments for noninvertible maps. In Section 3, we apply this result to Axiom A and nonuniformly hyperbolic diffeomorphisms. In Section 4, we consider suspensions flows subject to the requirement that the roof function is bounded above and below. This covers the case of Axiom A flows and many nonuniformly hyperbolic flows. In Section 5, we show how to relax the conditions on the roof 
functions following [3, 4], allowing us to treat Lorentz gases with cusps and Lorenz attractors.

\section{Convergence of moments for maps}

Let $(X, \mu)$ be a probability space and $f: X \rightarrow X$ an ergodic measure-preserving map. Let $L: L^{1}(X, \mu) \rightarrow L^{1}(X, \mu)$ be the transfer operator, defined by $\int_{X} L v w d \mu=$ $\int_{X} v w \circ f d \mu$ for all $w \in L^{\infty}(X, \mu)$. Given an observable $v: X \rightarrow \mathbb{R}$, define the Birkhoff sum $v_{n}=\sum_{j=0}^{n-1} v \circ f^{j}$.

Lemma 2.1 Let $p \geq 2$. Suppose that $v \in L^{p}(X, \mu)$ with $\int_{X} v d \mu=0$, and that $\sum_{j=1}^{\infty}\left\|L^{j} v\right\|_{p}<\infty$. Then

(a) The limit $\sigma^{2}=\lim _{n \rightarrow \infty} \int_{X}\left(n^{-\frac{1}{2}} v_{n}\right)^{2} d \mu$ exists.

(b) $\sigma^{2}=0$ if and only if $v=\chi \circ f-\chi$ for some $\chi \in L^{p}$.

(c) $n^{-\frac{1}{2}} v_{n} \rightarrow_{d} G$ as $n \rightarrow \infty$, where $G$ is normally distributed with mean zero and variance $\sigma^{2}$.

(d) There is a constant $C$ such that $\left\|n^{-\frac{1}{2}} v_{n}\right\|_{p} \leq C$.

(e) $\lim _{n \rightarrow \infty} \int_{X}\left|n^{-\frac{1}{2}} v_{n}\right|^{q} d \mu=E\left(|G|^{q}\right)$ for all $q<p$.

Proof Parts (a)-(c) are well-known and are included for completeness. Let $\chi=$ $\sum_{j=1}^{\infty} L^{j} v \in L^{p}$ and write $v=w+\chi \circ f-\chi$. A calculation shows that $L w=0$ and it follows that $\left\{w \circ f^{j}, j \geq 0\right\}$ is an orthogonal sequence in $L^{2}$. Hence $\int_{X}\left(w_{n}\right)^{2} d \mu=n \sigma^{2}$ where $\sigma^{2}=\int_{X} w^{2} d \mu$. Further,

$$
v_{n}=w_{n}+\chi \circ f^{n}-\chi
$$

and part (a) follows. Moreover, $\sigma^{2}=0$ if and only if $w=0$ proving (b).

Let $U v=v \circ f$ and note that $L$ is the $L^{2}$ adjoint of $U$. Hence $L U=I$ and it is standard that $U L=E\left(\cdot \mid f^{-1} \mathcal{M}\right)$ where $\mathcal{M}$ is the underlying $\sigma$-algebra on $X$. In particular, $E\left(w \mid f^{-1} \mathcal{M}\right)=0$. Following Gordin [19], we note that the sequence $\left\{w \circ f^{j}, j \geq 0\right\}$ is a reverse martingale increment sequence. (For more details see for example [18, Remark 3.12].) Passing to the inverse limit $\tilde{f}: \tilde{X} \rightarrow \tilde{X}$ and the lifted observable $\tilde{w}: \tilde{X} \rightarrow \mathbb{R}$, we obtain a forward martingale sequence $\left\{\tilde{w} \circ \tilde{f}^{-j}, j \geq 0\right\}$. By Billingsley [6], $n^{-\frac{1}{2}} \sum_{j=0}^{n} \tilde{w} \circ \tilde{f}^{-j} \rightarrow_{d} G$. By Burkholder's inequality [10], $\| \sum_{j=0}^{n-1} \tilde{w} \circ$ 
$\tilde{f}^{-j} \|_{p}=O\left(n^{\frac{1}{2}}\right)$. But $\sum_{j=0}^{n-1} \tilde{w} \circ \tilde{f}^{-j}=\left\{\sum_{j=0}^{n-1} \tilde{w} \circ \tilde{f}^{j}\right\} \circ \tilde{f}^{-n}={ }_{d} \sum_{j=0}^{n-1} \tilde{w} \circ \tilde{f}^{j}={ }_{d} w_{n}$ so it follows that $n^{-\frac{1}{2}} w_{n} \rightarrow{ }_{d} G$ and that $\left\|w_{n}\right\|_{p}=O\left(n^{\frac{1}{2}}\right)$. Hence parts (c) and (d) follow from (2.1).

Finally, by standard probability theory (eg. [17, Exercise 2.5, p. 86]), part (e) is a direct consequence of parts (c) and (d).

Usually we are interested in $L^{\infty}$ observables. As observed in [28], it is then possible to use an inequality due to Rio [35, Theorem 2.5] (see [30, Proposition 7]) to obtain an improved result on the order of converging moments.

Lemma 2.2 Assume the set up of Lemma 2.1 with $p \geq 2$, and suppose further that $v \in L^{\infty}$. Then

$\left(d^{\prime}\right)$ There is a constant $C$ such that $\left\|n^{-\frac{1}{2}} v_{n}\right\|_{2 p} \leq C$.

(e') $\lim _{n \rightarrow \infty} \int_{X}\left|n^{-\frac{1}{2}} v_{n}\right|^{q} d \mu=E\left(|G|^{q}\right)$ for all $q<2 p$.

Proof Part $\left(d^{\prime}\right)$ is proved in the course of the proof of [28, Theorem 3.1] (cf. [28, Equation (3.1)]. Again, part $\left(\mathrm{e}^{\prime}\right)$ is an elementary consequence of $\left(\mathrm{d}^{\prime}\right)$ and Lemma 2.1(c).

Remark 2.3 We have focused on the cases $v \in L^{p}$ and $v \in L^{\infty}$ since (i) the second result is sufficient for our applications and (ii) the first result is much simpler and yet suffices for most of the examples. However, the arguments easily extend to the case $v \in L^{a}$ for all $a \in[p, \infty]$. Assume the set up of Lemma 2.1 with $p \geq 2$, and suppose further that $v \in L^{a}, a \geq p$. Write $2 s^{-1}=p^{-1}+a^{-1}$. Then

$\left(\mathrm{d}^{\prime \prime}\right)$ There is a constant $C$ such that $\left\|n^{-\frac{1}{2}} v_{n}\right\|_{s} \leq C$.

$\left(\mathrm{e}^{\prime \prime}\right) \lim _{n \rightarrow \infty} \int_{X}\left|n^{-\frac{1}{2}} v_{n}\right|^{q} d \mu=E\left(|G|^{q}\right)$ for all $q<s$.

To see this define

$$
b_{i, n}=\max _{i \leq u \leq n}\left\|v \circ f^{i} \sum_{k=i}^{u} E\left(v \circ f^{k} \mid \mathcal{F}_{i}\right)\right\|_{s / 2},
$$

where $\mathcal{F}_{i}$ is the filtration associated with the process $v \circ f^{i}$. Rio's inequality implies that boundedness of $\left\|n^{-\frac{1}{2}} v_{n}\right\|_{s}$ follows from bounded of $b_{i, n}$. By Hölder's inequality

$$
b_{i, n} \leq\|v\|_{a} \max _{i \leq u \leq n}\left\|\sum_{k=i}^{u} E\left(v \circ f^{k} \mid \mathcal{F}_{i}\right)\right\|_{p},
$$

and boundedness follows as in [28]. 


\section{Uniformly and nonuniformly hyperbolic diffeo- morphisms}

We begin this section by recasting the main assumption of Lemmas 2.1 and 2.2 in terms of decay of correlations against a class of test functions. Such a criterion is equivalent but perhaps more familiar, and is standard in the literature for noninvertible dynamical systems.

Proposition 3.1 Let $p \in[1, \infty]$ and let $v: X \rightarrow \mathbb{R}$ be an $L^{p}$ observable with $\int_{X} v d \mu=0$. Suppose $\frac{1}{p}+\frac{1}{p^{\prime}}=1$. Then $\left\|L^{j} v\right\|_{p}=\sup \left|\int_{X} v w \circ f^{j} d \mu\right|$ where the supremum is over all $w \in L^{p^{\prime}}$ with $\|w\|_{p^{\prime}}=1$.

Proof Since $\int_{X} L^{j} v w d \mu=\int_{X} v w \circ f^{j} d \mu$, the result follows from the duality of $L^{p}$ and $L^{p^{\prime}}$.

Example 3.2 (Uniformly expanding maps) If $X$ is a mixing one-sided subshift of finite type and $v$ is Lipschitz with respect to a symbolic metric, then $v$ has exponential decay of correlations against all $L^{1}$ observables $w$ (see for example [31]), so that $\sum_{j=1}^{\infty}\left\|L^{j} v\right\|_{\infty}<\infty$. By Lemma 2.1, we obtain convergence of $q$ th moments for all $q$.

Example 3.3 (Nonuniformly expanding maps) The situation is slightly different for nonuniformly expanding (quotient) Young towers [42, 43]. Let $v$ be a Lipschitz observable (again with respect to a suitable symbolic metric). In the case of exponential tails [42], exponential decay of correlations holds against all $L^{\infty}$ observables. By Proposition 3.1, $\left\|L^{j} v\right\|_{1} \leq C e^{-a j}$ where $C=C(v)>0, a>0$. Hence $\int_{X}\left|L^{j} v\right|^{p} d \mu \leq\left\|L^{j} v\right\|_{\infty}^{p-1}\left\|L^{j} v\right\|_{1} \leq\|v\|_{\infty}^{p-1}\left\|L^{j} v\right\|_{1}$ so $\left\|L^{j} v\right\|_{p} \leq\|v\|_{\infty}^{1-\frac{1}{p}} C^{\frac{1}{p}} e^{-(a / p) j}$ which is summable for all $p<\infty$. By Lemma 2.1, we again obtain convergence of $q$ th moments for all $q$.

In the case of polynomial tails $\mu(r>n)=O\left(n^{-(\beta+1)}\right), \beta>1$, Young [43] proved that $v$ has polynomial decay of correlations against $L^{\infty}$ observables and further $\left\|L^{j} v\right\|_{1} \leq C j^{-\beta}$. (This correlation decay rate is optimal by Sarig [37] and Gouëzel [20].) The same interpolation argument as in the exponential case shows that $\left\|L^{j} v\right\|_{p} \leq\|v\|_{\infty}^{1-\frac{1}{p}} C^{\frac{1}{p}} j^{-\beta / p}$ which is summable for all $p<\beta$. By Lemma 2.2, we obtain convergence of $q$ th moments for all $q<2 \beta$.

Theorem 3.4 (Uniformly hyperbolic diffeomorphisms) Suppose that $\Lambda$ is a hyperbolic basic set for an Axiom A diffeomorphism $f$. We suppose that $\Lambda$ is nontrivial (not a periodic orbit). Let $\mu$ be the equilibrium measure corresponding to a 
fixed Hölder potential. Let $v: \Lambda \rightarrow \mathbb{R}$ be a Hölder observable with $\int_{\Lambda} v d \mu=0$. Then $n^{-\frac{1}{2}} v_{n} \rightarrow_{d} G$ where $G$ is normal with mean zero and variance $\sigma^{2} \geq 0$, and $\lim _{n \rightarrow \infty} \int_{\Lambda}\left|n^{-\frac{1}{2}} v_{n}\right|^{q} d \mu=E\left(|G|^{q}\right)$ for all $q \geq 0$.

Proof We use a number of standard facts about hyperbolic basic sets (see for example [31]). Basic sets are mixing up to a finite cycle, so it is easy to see that we may suppose without loss that $f: \Lambda \rightarrow \Lambda$ is mixing. By Bowen [7], $\Lambda$ can be modelled by a subshift of finite type, and $v$ lifts to an observable (which we also denote by $v$ ) that is Lipschitz with respect to a suitable symbolic metric. An argument of Sinai [39] shows that $v$ is cohomologous to an observable that depends only on future coordinates and hence is well-defined on the associated one-sided subshift of finite type. The cobounding function is Lipschitz (with respect to a weaker symbolic metric) and in particular is in $L^{\infty}$.

After these reductions, we may suppose without loss that $f: \Lambda \rightarrow \Lambda$ is a mixing one-sided subshift of finite type and that $v: \Lambda \rightarrow \mathbb{R}$ is a Lipschitz observable of mean zero. We are now in the situation of Example 3.2 and the result follows from Lemma 2.1.

Next, we consider the case when $f: \Lambda \rightarrow \Lambda$ is a nonuniformly hyperbolic map modelled by a Young tower $[42,43]$. Roughly speaking, a Young tower $f: \Delta \rightarrow \Delta$ is a discrete suspension of a "uniformly hyperbolic" base map $F: Y \rightarrow Y$ with an integrable return time function $r: Y \rightarrow \mathbb{Z}^{+}($so $\Delta=Y \times \mathbb{Z} / \sim$ where $(y, r(y)) \sim$ $(F y, 0)$ and $f(y, \ell)=(y, \ell+1)$ computed modulo $\sim)$. Invariant probability measures $\mu$ on $\Delta$ and $\mu_{Y}$ on $Y$ are related by $\mu=\left(\mu_{Y} \times\right.$ counting $) / \bar{r}$ where $\bar{r}=\int_{Y} r d \mu_{Y}$.

Theorem 3.5 (Nonuniformly hyperbolic maps) Suppose that $\Lambda$ is a nonuniformly hyperbolic map modelled by a Young tower with return time $r \in L^{2}\left(Y, \mu_{Y}\right)$ (equivalently $n \mu_{Y}(r>n)$ is summable). Let $v: \Lambda \rightarrow \mathbb{R}$ be a Hölder observable with $\int_{\Lambda} v d \mu=0$. Then

(a) $n^{-\frac{1}{2}} v_{n} \rightarrow_{d} G$ where $G$ is normal with mean zero and variance $\sigma^{2} \geq 0$.

(b) If $\mu_{Y}(r>n)$ decays exponentially, then $\lim _{n \rightarrow \infty} \int_{\Lambda}\left|n^{-\frac{1}{2}} v_{n}\right|^{q} d \mu=E\left(|G|^{q}\right)$ for all $q \geq 0$.

(c) If $\mu_{Y}(r>n)=O\left(n^{-(\beta+1)}\right), \beta>1$, then $\lim _{n \rightarrow \infty} \int_{\Lambda}\left|n^{-\frac{1}{2}} v_{n}\right|^{q} d \mu=E\left(|G|^{q}\right)$ for all $q \in[0,2 \beta)$.

Proof As in the proof of Theorem 3.4 we can make a number of simplifying steps. Young towers are mixing up to a finite cycle, so we may suppose without loss that $f$ 
is mixing. A version of the Sinai argument [27, Lemma 3.2] shows that the observable is cohomologous to a Lipschitz observable that depends only on future coordinates, and the cobounding function is in $L^{\infty}$. Hence we can pass to a quotient tower which is nonuniformly expanding.

We are now in the situation of Example 3.3. Parts (a) and (b) follow from Lemma 2.1 and part (c) follows from Lemma 2.2.

Example 3.6 (Nonuniformly hyperbolic maps) Part (b) of Theorem 3.5 includes Hénon-like attractors [5], and planar periodic dispersing billiards with both finite [42] and infinite [11] horizon.

Theorem 3.5(c) is illustrated by Pomeau-Manneville intermittency maps [33]. In particular, the example of [23] is a Markov map with an indifferent fixed point at 0 and $f x \approx x^{1+\alpha}, \alpha \in(0,1)$. These maps can be modelled by Young towers with $\mu_{Y}(r>n) \approx n^{-(\beta+1)}$ where $\beta=\alpha^{-1}-1$. If $\alpha \in\left(0, \frac{1}{2}\right)$ then we can apply Theorem 3.5 to obtain convergence of all moments of order $q<2 \beta$.

More generally, Zweimüller [44, 45] studied a class of non-Markovian maps with indifferent fixed points (called AFN maps), and our results apply to these maps when the return time function $r$ is square-integrable.

Bunimovich flowers [9] are a class of billiards modelled by Young towers with polynomial tails and $\beta=2$ (at least up to a logarithmic term) [14]. By Theorem 3.5(c) we obtain convergence of $q$ th moments for $q<4$.

Chernov \& Zhang [13] introduced a class of planar periodic dispersing billiards with scatterers having points of vanishing curvature. Such billiards are modelled by Young towers with polynomial tails, and every value of $\beta \in(2, \infty)$ is achieved. These examples are covered by Theorem 3.5(c).

Remark 3.7 Our result on the order of converging moments is essentially optimal. Consider the example in [28] where $v \equiv 1$ on the right-hand-side of a Young tower with $\mu_{Y}(r>n) \sim n^{-(\beta+1)}$. More precisely, $v$ is taken to be a mean zero observable satisfying $v(y, \ell)=1$ for all $(y, \ell)$ with $0 \leq \ell<r(y)$ and $r(y)>N_{0}$ for some fixed $N_{0}$. Then $\mu\left(v_{n}=n\right) \geq c n^{-\beta}$ where $c>0$. Hence $\left\|n^{-\frac{1}{2}} v_{n}\right\|_{q} \geq c^{1 / q} n^{1 / 2} n^{-\beta / q}$ which diverges for $q>2 \beta$.

\section{Uniformly and nonuniformly hyperbolic flows}

Let $(X, \mu)$ be a probability space and $f: X \rightarrow X$ an ergodic measure preserving transformation. Given an integrable roof function $h: X \rightarrow \mathbb{R}^{+}$, we form the suspension $X^{h}=X \times \mathbb{R} / \sim$ where $(x, h(x)) \sim(f x, 0)$. The suspension flow is given by 
$f_{t}(x, u)=(x, u+t)$ computed modulo $\sim$, with ergodic invariant probability measure $\mu^{h}=(\mu \times$ Lebesgue $) / \bar{h}$ where $\bar{h}=\int_{X} h d \mu$.

Let $\phi: X^{h} \rightarrow \mathbb{R}$ be an $L^{1}$ observable with $\int_{X^{h}} \phi d \mu^{h}=0$. We define $\phi_{t}=$ $\int_{0}^{t} \phi \circ f_{s} d s$. Also, we define the induced observable $\Phi(x)=\int_{0}^{h(x)} \phi(x, u) d u$, and as usual set $\Phi_{n}=\sum_{j=0}^{n-1} \Phi \circ f^{n}$.

Lemma 4.1 Assume that $h, h^{-1} \in L^{\infty}(X, \mu)$. Let $p>2$ and $\gamma \in\left[\frac{1}{2}, 1\right]$. Let $\phi$ : $X^{h} \rightarrow \mathbb{R}$ be an observable in $L^{p}\left(X^{h}\right)$ with $\int_{X^{h}} \phi d \mu^{h}=0$, and define $\Phi$ as above. (In particular, $\Phi \in L^{p}(X, \mu)$.) If $\left\|\Phi_{n}\right\|_{L^{p}(X, \mu)}=O\left(n^{\gamma}\right)$, then $\left\|\phi_{t}\right\|_{L^{p}\left(X^{h}\right)}=O\left(t^{\gamma}\right)$.

Proof Passing to the natural extension if necessary, we may suppose without loss that $f$ is invertible. Set $\Phi_{-n}=\sum_{j=0}^{n-1} \Phi \circ f^{-j}$ for $n \geq 0$.

Define the lap number $N[\cdot, t]$ to be the largest integer $n$ such that $h_{n}(x) \leq t$. By [29, Remark 2.2], $\phi_{t}(x, u)=\Phi_{N[x, t]}(x)+O(1)$ uniformly in $(x, u) \in X^{h}, t>0$. Note that $\Phi_{N[\cdot, t]}$ can be regarded as a function on $X^{h}$ (independent of the second variable) or as a function on $X$. Such a function $g(x, u)=\tilde{g}(x)$ has the property that $\|g\|_{L^{p}\left(X^{h}, \mu^{h}\right)} \leq \bar{h}^{-1 / p}\|h\|_{\infty}^{1 / p}\|\tilde{g}\|_{L^{p}(X, \mu)}$. Hence it suffices to show that $\left\|\Phi_{N[\cdot, t]}\right\|_{p}=O\left(t^{\gamma}\right)$ where from now on $L^{p}$ means $L^{p}(X, \mu)$.

By assumption, $\left\|\Phi_{n}\right\|_{p}=O\left(n^{\gamma}\right)$ and hence by Serfling [38, Corollary B1],

$$
\left\|\max _{|j| \leq n}\left|\Phi_{j}\right|\right\|_{p}=O\left(n^{\gamma}\right)
$$

Since $h$ is bounded below, $|N[\cdot, t]| \leq$ at where $a=\left\|h^{-1}\right\|_{\infty}^{-1}$. Hence $\left\|\Phi_{N[\cdot, t]}\right\|_{p} \leq$ $\left\|\max _{|j| \leq a t}\left|\Phi_{j}\right|\right\|_{p}=O\left(t^{\gamma}\right)$ as required.

Corollary 4.2 Assume the hypotheses of Lemma 4.1 and suppose that $t^{-\gamma} \phi_{t} \rightarrow{ }_{d} G$ on $\left(X^{h}, \mu^{h}\right)$ for some random variable $G$. Then $\lim _{t \rightarrow \infty} \int_{X^{h}}\left|t^{-\gamma} \phi_{t}\right|^{q} d \mu^{h}=E\left(|G|^{q}\right)$ for all $q<p$.

Proof Again we can apply [17, Exercise 2.5, p. 86]), so this is an immediate consequence of Lemma 4.1 .

Example 4.3 (Uniformly hyperbolic flows) Suppose that $\Lambda$ is a nontrivial hyperbolic set for an Axiom A flow $f_{t}$. Then $\Lambda$ can be modelled as a suspension $X^{h}$ of a subshift of finite type with Lipschitz roof function $h: X \rightarrow \mathbb{R}^{+}$bounded above and below. Let $\mu$ be an equilibrium measure on $X$ (corresponding to a Lipschitz potential) and construct $\mu^{h}$ on $X^{h}$, with corresponding measure $\nu$ on $\Lambda$. Let $\phi: \Lambda \rightarrow \mathbb{R}$ be a Hölder observable with $\int_{\Lambda} \phi d \nu=0$. By [34, 29], $t^{-\frac{1}{2}} \phi_{t} \rightarrow{ }_{d} G$ where $G$ is normal 
with mean zero and variance $\sigma^{2} \geq 0$. By Theorem 3.4 and Corollary 4.2 we obtain that $\lim _{n \rightarrow \infty} \int_{\Lambda}\left|n^{-\frac{1}{2}} v_{n}\right|^{q} d \nu=E\left(|G|^{q}\right)$ for all $q \geq 0$.

Example 4.4 (Nonuniformly hyperbolic flows) Similarly our results apply for certain nonuniformly expanding/hyperbolic flows, namely those that can be regarded as suspension flows over Young towers with Hölder roof functions that are bounded above and below. Provided the return time function $r$ for the Young tower is square integrable, it follows from [29] that the CLT holds for Hölder observables.

If $r$ has exponential (or at least superpolynomial) tails, then $q$ th moments converge for all $q \geq 0$. This includes finite horizon planar periodic Lorentz gases and suspended Hénon-like attractors.

In the case of polynomial tails $\mu_{Y}(r>n)=O\left(n^{-(\beta+1)}\right)$, we obtain convergence of $q$ th moments for all $q<2 \beta$. This applies for instance to the Lorentz gas flow corresponding to the examples of Chernov \& Zhang [13] described in Example 3.6.

\section{$5 \quad$ More general flows}

In Section 4, we assumed that the roof function $h$ satisfies $h, h^{-1} \in L^{\infty}(X)$. We now discuss how to relax these assumptions.

Suppose that $f: X \rightarrow X$ is nonuniformly hyperbolic (modelled by a Young tower) and that $h: X \rightarrow \mathbb{R}^{+}$is a Hölder roof function. (More generally, we allow $h$ to have discontinuities provided that $h$ is in $L^{\infty}(X)$ and is piecewise Hölder on partition elements of the Young tower with uniform Hölder constant.) As shown in Bálint \& Melbourne [3], when $h$ is not bounded below a useful approach is to choose a new cross-section $X^{\prime}$ to the flow with modified roof function $h^{\prime}$ such that $X^{h}=\left(X^{\prime}\right)^{h^{\prime}}$. If this is done in such a way that $h^{\prime}$ is (piecewise) Hölder and $h^{\prime},\left(h^{\prime}\right)^{-1} \in L^{\infty}\left(X^{\prime}\right)$ then Lemma 4.1 and Corollary 4.2 may apply.

Example 5.1 (Lorentz gases with cusps) For example, consider a Sinai billiard with cusps. Then the billiard map $X^{h}$ is slowly mixing with $\beta=1[12,15]$, and Theorem 3.5 does not apply. Moreover, $h$ is not bounded below. However there is an alternative cross-section $X^{\prime}$ bounded away from the cusps such that the Poincare map $f^{\prime}: X^{\prime} \rightarrow X^{\prime}$ is modelled by a Young tower with exponential tails. Theorem 3.5 applies to the moments for this modified map. More importantly, the roof function $h^{\prime}$ can be shown to be bounded above and below [3]. In this way, [3] proved the CLT (and almost sure invariance principle) for the Lorentz flow with cusps. By Corollary 4.2, $q$ th moments converge for the flow for all $q<\infty$. 
Example 5.2 (Bunimovich flowers) A second example considered in [3] is Bunimovich flowers. Here the billiard map $f: X \rightarrow X$ is mixing with $\beta=2$ up to a logarithmic factor (see Example 3.6) so moments converge for the map for $q<4$. Again, the roof function $h$ is not bounded below, so Corollary 4.2 does not apply directly. However, there is a different choice of Poincaré map $f^{\prime}: X^{\prime} \rightarrow X^{\prime}$ with the same value of $\beta$. In addition $h^{\prime}$ is bounded above and below, and moments converge for the flow for $q<4$.

There are many ways to deal with unbounded roof functions, for example (i) modify the construction of the Young tower [22] so as to keep control of the unboundedness of $h$, (ii) approximate the dynamics by a truncated tower on which the roof function is bounded [26]. An alternative approach of Bálint \& Melbourne [4] is to work with a fixed tower model, but to modify the return time function $r$ of the tower and the roof function $h$ of the flow in such a way that the Poincaré map to the base $Y$ of the tower is unchanged. More precisely, define the induced roof function $H: Y \rightarrow \mathbb{R}^{+}$ by $H(y)=\sum_{\ell=0}^{r(y)-1} h(y, \ell)$. Define modified versions $r^{\prime}$ and $h^{\prime}$ of $r$ and $h$ in such a way that $H^{\prime}=H$. Then the modified suspension flow is still an extension of the original flow. The aim is to do this in such a way that $h^{\prime}$ is (piecewise) Hölder and $h^{\prime}$, $\left(h^{\prime}\right)^{-1} \in L^{\infty}(X)$. This approach seems to give optimal results in situations where the other methods do not (for instance, decay of correlations for infinite horizon Lorentz gases [4]).

Example 5.3 (Geometric Lorenz attractors) The geometric Lorenz attractor $[1,21]$ can be modelled as a suspension flow over a Young tower with exponential tails, but the roof function $h$ is unbounded with a logarithmic singularity. By [4], the return time function $r$ and roof function $h$ can be modified in such a way that the Poincaré map to the base of the tower is unchanged, and the modified roof function $h^{\prime}$ is (piecewise) Hölder with $h^{\prime},\left(h^{\prime}\right)^{-1} \in L^{\infty}(X)$. The modified return tails $\mu_{Y}\left(r^{\prime}>n\right)$ decay at a stretched exponential rate. This recovers the CLT (and almost sure invariance principle) proved by [22]. By Example 4.4, qth moments converge for the flow for all $q<\infty$.

By Tucker [41], our result applies in particular to the classical Lorenz equations [24].

Acknowledgements This research of IM was supported in part by EPSRC Grant EP/F031807/1. This research of AT was supported in part by NSF Grant DMS0607345 . 


\section{References}

[1] V. S. Afraĭmovič, V. V. Bykov and L. P. Silnikov. The origin and structure of the Lorenz attractor. Dokl. Akad. Nauk SSSR 234 (1977) 336-339.

[2] D. N. Armstead, B. R. Hunt and E. Ott. Anomalous diffusion in infinite horizon billiards. Phys. Rev. E 67 (2003) 021110.

[3] P. Bálint and I. Melbourne. Decay of correlations and invariance principles for dispersing billiards with cusps, and related planar billiard flows. J. Stat. Phys. 133 (2008) 435-447.

[4] P. Bálint and I. Melbourne. Decay of correlations for flows with unbounded roof function, including the infinite horizon planar periodic Lorentz gas. Preprint, November 2010.

[5] M. Benedicks and L.-S. Young. Markov extensions and decay of correlations for certain Hénon maps. Astérisque (2000) no. 261, 13-56.

[6] P. Billingsley. The Lindeberg-Lévy theorem for martingales. Proc. Amer. Math. Soc. 12 (1961) 788-792.

[7] R. Bowen. Markov partitions for Axiom A diffeomorphisms. Amer. J. Math. 92 (1970) 725-747.

[8] R. Bowen. Equilibrium States and the Ergodic Theory of Anosov Diffeomorphisms. Lecture Notes in Math. 470, Springer, Berlin, 1975.

[9] L. A. Bunimovič. The ergodic properties of billiards that are nearly scattering. Dokl. Akad. Nauk SSSR 211 (1973) 1024-1026.

[10] D. L. Burkholder. Distribution function inequalities for martingales. Ann. Probability 1 (1973) 19-42.

[11] N. Chernov. Decay of correlations and dispersing billiards. J. Statist. Phys. 94 (1999) 513-556.

[12] N. Chernov and R. Markarian. Dispersing billiards with cusps: slow decay of correlations. Comm. Math. Phys. 270 (2007) 727-758.

[13] N. Chernov and H.-K. Zhang. A family of chaotic billiards with variable mixing rates. Stoch. Dyn. 5 (2005) 535-553.

[14] N. I. Chernov and H.-K. Zhang. Billiards with polynomial mixing rates. Nonlinearity 18 (2005) 1527-1553. 
[15] N. I. Chernov and H.-K. Zhang. Improved estimates for correlations in billiards. Commun. Math. Phys. 77 (2008) 305-321.

[16] M. Courbage, M. Edelman, S. M. Saberi Fathi and G. M. Zaslavsky. Problem of transport in billiards with infinite horizon. Phys. Rev. E 77 (2008) 036203.

[17] R. Durrett. Probability: theory and examples, third ed., Duxbury Press, Belmont, CA, 1996.

[18] M. J. Field, I. Melbourne and A. Török. Decay of correlations, central limit theorems and approximation by Brownian motion for compact Lie group extensions. Ergodic Theory Dynam. Systems 23 (2003) 87-110.

[19] M. I. Gordin. The central limit theorem for stationary processes. Soviet Math. Dokl. 10 (1969) 1174-1176.

[20] S. Gouëzel. Sharp polynomial estimates for the decay of correlations. Israel J. Math. 139 (2004) 29-65.

[21] J. Guckenheimer and R. F. Williams. Structural stability of Lorenz attractors. Inst. Hautes Études Sci. Publ. Math. 50 (1979) 59-72.

[22] M. Holland and I. Melbourne. Central limit theorems and invariance principles for Lorenz attractors. J. London Math. Soc. 76 (2007) 345-364.

[23] C. Liverani, B. Saussol and S. Vaienti. A probabilistic approach to intermittency. Ergodic Theory Dynam. Systems 19 (1999) 671-685.

[24] E. D. Lorenz. Deterministic nonperiodic flow. J. Atmosph. Sci. 20 (1963) 130141.

[25] C. McMullen. Thermodynamics, dimension and the Weil-Petersson metric. Invent. Math. 173 (2008) 365-425.

[26] I. Melbourne. Decay of correlations for slowly mixing flows. Proc. London Math. Soc. 98 (2009) 163-190.

[27] I. Melbourne and M. Nicol. Almost sure invariance principle for nonuniformly hyperbolic systems. Commun. Math. Phys. 260 (2005) 131-146.

[28] I. Melbourne and M. Nicol. Large deviations for nonuniformly hyperbolic systems. Trans. Amer. Math. Soc. 360 (2008) 6661-6676.

[29] I. Melbourne and A. Török. Statistical limit theorems for suspension flows. Israel J. Math. 144 (2004) 191-209. 
[30] F. Merlevède, M. Peligrad and S. Utev. Recent advances in invariance principles for stationary sequences. Probab. Surv. 3 (2006) 1-36 (electronic).

[31] W. Parry and M. Pollicott. Zeta Functions and the Periodic Orbit Structure of Hyperbolic Dynamics. Astérique 187-188, Société Mathématique de France, Montrouge, 1990.

[32] M. Pollicott. On the rate of mixing of Axiom A flows. Invent. Math. 81 (1985) 413-426.

[33] Y. Pomeau and P. Manneville. Intermittent transition to turbulence in dissipative dynamical systems. Comm. Math. Phys. 74 (1980) 189-197.

[34] M. Ratner. The central limit theorem for geodesic flows on $n$-dimensional manifolds of negative curvature. Israel J. Math. 16 (1973) 181-197.

[35] E. Rio. Théorie asymptotique des processus aléatoires faiblement dépendants. Mathématiques \& Applications (Berlin) [Mathematics \& Applications] 31, Springer-Verlag, Berlin, 2000.

[36] D. Ruelle. Thermodynamic Formalism. Encyclopedia of Math. and its Applications 5, Addison Wesley, Massachusetts, 1978.

[37] O. M. Sarig. Subexponential decay of correlations. Invent. Math. 150 (2002) $629-653$.

[38] R. J. Serfling. Moment inequalities for the maximum cumulative sum. Ann. Math. Statist. 41 (1970) 1227-1234.

[39] Y. G. Sinal̆. Gibbs measures in ergodic theory. Russ. Math. Surv. 27 (1972) 21-70.

[40] D. Szász and T. Varjú. Limit laws and recurrence for the planar Lorentz process with infinite horizon. J. Stat. Phys. 129 (2007) 59-80.

[41] W. Tucker. A rigorous ODE solver and Smale's 14th problem. Found. Comput. Math. 2 (2002) 53-117.

[42] L.-S. Young. Statistical properties of dynamical systems with some hyperbolicity. Ann. of Math. 147 (1998) 585-650.

[43] L.-S. Young. Recurrence times and rates of mixing. Israel J. Math. 110 (1999) $153-188$. 
[44] R. Zweimüller. Ergodic structure and invariant densities of non-Markovian interval maps with indifferent fixed points. Nonlinearity 11 (1998) 1263-1276.

[45] R. Zweimüller. Ergodic properties of infinite measure-preserving interval maps with indifferent fixed points. Ergodic Theory Dynam. Systems 20 (2000) 15191549 . 